\title{
Publisher Correction: Chromatin dysregulation and DNA methylation at transcription start sites associated with transcriptional repression in cancers
}

\author{
Mizuo Ando (1) 1,2, Yuki Saito1,2, Guorong Xu³, Nam Q. Bui (10 4,5, Kate Medetgul-Ernar ${ }^{1}$, Minya Pu1',

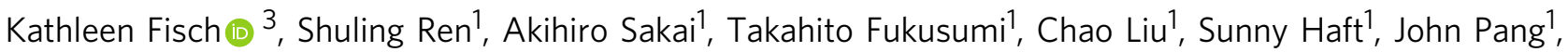 \\ Adam Mark ${ }^{3}$, Daria A. Gaykalova ${ }^{6}$, Theresa Guo ${ }^{6}$, Alexander V. Favorov (10 7,8, Srinivasan Yegnasubramanian ${ }^{7}$, \\ Elana J. Fertig7, Patrick Ha (10 ${ }^{9}$, Pablo Tamayo (1) ${ }^{1}$, Tatsuya Yamasoba (1) ${ }^{2}$, Trey Ideker ${ }^{4}$, Karen Messer ${ }^{1} \&$ \\ Joseph A. Califano ${ }^{1,10}$
}

Correction to: Nature Communications https://doi.org/10.1038/s41467-019-09937-w, published online 16 May 2019.

The original version of this Article contained an error in the author affiliations.

Trey Ideker was incorrectly associated with 'Department of Medicine (Oncology), Stanford University School of Medicine, 875 Blake Wilbur Dr, Palo Alto, CA 94304, USA.'

This has now been corrected in both the PDF and HTML versions of the Article.

Published online: 29 May 2019

\begin{abstract}
(c) (1) Open Access This article is licensed under a Creative Commons Attribution 4.0 International License, which permits use, sharing, adaptation, distribution and reproduction in any medium or format, as long as you give appropriate credit to the original author(s) and the source, provide a link to the Creative Commons license, and indicate if changes were made. The images or other third party material in this article are included in the article's Creative Commons license, unless indicated otherwise in a credit line to the material. If material is not included in the article's Creative Commons license and your intended use is not permitted by statutory regulation or exceeds the permitted use, you will need to obtain permission directly from the copyright holder. To view a copy of this license, visit http://creativecommons.org/licenses/by/4.0/.
\end{abstract}

() The Author(s) 2019

\footnotetext{
${ }^{1}$ Moores Cancer Center, University of California San Diego, 3855 Health Sciences Dr, La Jolla, CA 92093, USA. ${ }^{2}$ Department of Otolaryngology - Head and Neck Surgery, Graduate School of Medicine, University of Tokyo, 7-3-1 Hongo, Bunkyo-ku, Tokyo 113-8655, Japan. ${ }^{3}$ Department of Medicine, Center for Computational Biology and Bioinformatics, University of California San Diego, 9500 Gilman Drive, La Jolla, CA 92093, USA. ${ }^{4}$ Department of Medicine, University of California San Diego, 9500 Gilman Drive, La Jolla, CA 92093, USA. 5 Department of Medicine (Oncology), Stanford University School of Medicine, 875 Blake Wilbur Dr, Palo Alto, CA 94304, USA. ${ }^{6}$ Department of Otolaryngology - Head and Neck Surgery, Johns Hopkins University School of Medicine, 601 N Caroline St, Baltimore, MD 21287, USA. 7 Department of Oncology, Sidney Kimmel Comprehensive Cancer Center, Johns Hopkins University School of Medicine, $401 \mathrm{~N}$ Broadway, Baltimore, MD 21231, USA. ${ }^{8}$ Laboratory of Systems Biology and Computational Genetics, Vavilov Institute of General Genetics, Russian Academy of Sciences, Gubkina str. 3, Moscow 119333 , Russia. ${ }^{9}$ Department of Otolaryngology - Head and Neck Surgery, University of California San Francisco, 2380 Sutter St, San Francisco, CA 94115, USA. 10 Division of Otolaryngology - Head and Neck Surgery, Department of Surgery, University of California San Diego, 9300 Campus Point Drive, La Jolla, CA 92037, USA. Correspondence and requests for materials should be addressed to J.A.C. (email: jcalifano@ucsd.edu)
} 